\title{
Usefulness of Echocardiography for General Surgery Patients on Intensive Care Units: When and How?
}

\author{
Jai-Hyun Chung, Namryeol Kim * \\ Department of Surgery, Korea University College of Medicine, Seoul, Korea
}

\section{Article history:}

Received: October 5, 2020

Revised: November 17, 2020

Accepted: November 18, 2020
*Corresponding Author:

Namryeol Kim

Department of Surgery, 148 Gurodong-Ro,

Guro-Gu, Seoul, Korea

E-mail: drknr304@outlook.com

\section{ORCID}

Namryeol Kim

https://orcid.org/0000-0003-0137-6125

Jai-Hyun Chung

https://orcid.org/0000-0002-0889-5609

\section{ABSTRACT}

Purpose: Echocardiography is useful in the management of critically ill patients, but there are few studies on the usefulness of an echocardiography for critical general surgery patients. This study aimed to address this by analyzing the indications and findings for and clinical outcomes between (1) chest trauma; (2) cardiac specific pathology; and (3) hemodynamic instability groups of critical general surgery patients

Methods: Retrospective analysis was performed on the medical records data from the last 5 years of general surgery patients who were admitted to the intensive care unit (ICU) and had an echocardiography at least once during their ICU stay. The patient data were divided into 3 groups according to the indication for an echocardiography and based on the clinical situation. Demographics, the ratio of positive echocardiography findings, and clinical outcomes including the length of hospital stay, length of ICU stay, and in-hospital mortality were analyzed among these groups.

Results: There were statistical differences in the rate of positive echocardiography findings $(p=$ $0.018)$ and in-hospital mortality $(p=0.001)$ amongst the groups with different echocardiography indications; there was no difference in the length of stay. Patients who had an echocardiography due to hemodynamic instability or cardiologic pathology presented with a higher ratio of positive echocardiography findings and mortality, compared with the chest trauma patient group. The chest trauma group showed the lowest rate of positive echocardiography findings (7\%).

Conclusion: Echocardiographies are useful for general surgery patients in ICUs when there are indications such as previous cardiac pathology history or hemodynamic instability.

Keywords: echocardiography, intensive care unit, general surgery

\section{Introduction}

Echocardiography has been a useful diagnostic tool and integral part of clinical cardiology, with applications in diagnosis, decision making, and management for patients with a wide range of cardiovascular diseases. This examination has been traditionally requested for patients who have a history of heart disease, variable abnormal findings from the basic physical examinations and studies such as auscultation, electrocardiography, chest $X$ rays and some laboratory tests, symptoms such as chest pain, respiratory distress, and even nonspecific fever, and bacteremia [1].
Recently, echocardiographies have been reported to be a valuable diagnostic and management tool in the intensive care unit (ICU) and emergency setting, beyond the previous traditional indications such as advanced diagnostic processes after initial basic cardiac studies [2]. Clinical manifestation of cardiovascular disease in ICU is diverse therefore, it has been challenging to develop methodical indications for an echocardiography for critically ill patients, so the decision to perform this examination tends to heavily depend on the physician's experience and intuition. However, the Society of Critical Care Medicine in 2016 recommended the use of echocardiography in ICU and produced guidelines for 
performing bedside echocardiographies in the ICU [3]. The usefulness of an echocardiography in the critical care field may be due to its merits of speed and ease of use, non-invasiveness type of examination, easily repeatable echocardiographies, performed at the bedside, and simultaneous evaluation of the structural and functional abnormality of the heart. With these distinct strengths, the use of echocardiographies in ICU have been increasing gradually [4].

It has been recently reported that significant cardiac pathologies were detected in $76.6 \%$ of echocardiographies performed on patients in ICU, with the most common cardiac pathology reported to be a systolic pulmonary arterial pressure $>50 \mathrm{mmHg}$, but these commonly observed cardiac abnormalities were not well considered in the current 2016 recommendations [5].

Indications for an echocardiographies in ICU are generally different from those in outpatient settings and include differential diagnosis of patients in hemodynamic shock [6], assessment of responsiveness to resuscitation, assessing cardiac function and wall motion in acute myocardial infarction or after recovery from cardiac arrest due to critical cardiac arrhythmia, monitoring of hemodynamic state with parameters such as cardiac output, and severe chest injury including cardiac injury [7]. In addition, echocardiographies are also used in evaluations of cardiac tamponade, pericardial effusion, endocarditis, and pulmonary embolism.

Many of the studies on echocardiographies target all patients in ICU (typically medical and cardiac surgery patients) and have demonstrated the usefulness of echocardiographies in critical care. However, studies on indications for echocardiographies on general surgery patients in ICU are rare to find. The purpose of this study was to address this and determine the effectiveness and indications in general surgery patients in ICU.

For this purpose, general surgery patient data was obtained from a single institution from patients who were admitted to ICU and had an echocardiography. The indications for echocardiography were classified into 3 simplified categories depending on the clinical situation: (1) chest trauma; (2) cardiac specific pathology based on previous history or basic study; and (3) hemodynamic instability. Then statistical association of clinical outcome parameters between each indication group were analyzed.

\section{Materials and Methods}

\section{Patient data collection}

All general surgery patients aged $\geq 18$ years who were admitted to the ICU of a single university hospital and had undergone a transthoracic echocardiography (TTE) at least once during their ICU stay from September 2014 to September
2019 were identified. The electronic medical records of these patients were retrospectively reviewed.

Data on demographic characteristics, clinical characteristics such as reason for hospital admission, appliance of mechanical ventilation and extracorporeal membrane oxygenation (ECMO), and the number of echocardiographic examinations received was obtained.

For comorbidity, only the following were included: hypertension, chronic obstructive pulmonary disease, coronary disease, renal disease, diabetes mellitus, and hepatitis.

The reasons for hospital admission were categorized as trauma, acute abdomen, malignancy, and benign disease. The acute abdomen group was defined as a group of patients who had undergone emergency abdominal surgery with pathologies including malignancy and with the exception of trauma. The trauma group included patients who were admitted to hospital due to injury regardless of whether they had undergone surgery or not. Patients undergoing emergency surgery due to injury were classified into the trauma group, not the acute abdomen group.

Indication for ICU admission was categorized as respiratory distress, hemodynamic instability, and close monitoring. The respiratory distress group was defined as requiring an oxygen supply and respiratory support with a high flow nasal cannula or use of mechanical ventilation. The hemodynamic instability group was defined as having a systolic blood pressure of $>90 \mathrm{mmHg}$ and a high clinical suspicion of shock, with features such as increased lactic acid level, altered mentality, and sudden increase in demand for inotropes/ vasopressors. Patients who were showing clinical features of circulatory shock and also needed concurrent mechanical ventilation were assigned to the hemodynamic instability group. The close monitoring group mostly consisted of postoperative patients with high preoperative risk assessed by formal interdepartmental consultations or preoperative visit decisions by anesthesiologists, and patients with a loss of consciousness or seizures. However, if the patients required mechanical ventilation or needed inotropes/vasopressors they were reclassified into the respiratory distress group or the hemodynamic instability group.

\section{Performance of echocardiography}

Cases of TTE were included and transesophageal echocardiography (TEE) examinations were excluded. All TTE examinations were performed at the bedside by 1 echocardiographer using TTE machines [Vivid i (GE Healthcare, Horten, Norway) or CX50 (Philips ultrasound, Bothell, WA)] upon the surgeon's request, usually on the same day as the request, and the results of the echocardiography were interpreted by 2 cardiologists. 


\section{Indications for echocardiography}

The indication for TTE was usually not stated by the surgeon who had requested it, but rather had to be inferred from a review of the medical records. The indications for TTE were categorized as (1) chest trauma; (2) cardiac specifics; and (3) hemodynamic instability. Chest trauma was defined as an Abbreviated Injury Scale (AIS) $\geq 3$ on the chest region regardless of any other associated injury. The patients in the chest trauma group had mainly multiple rib fractures with or without flail, hemopneumothorax, or lung contusions. Cardiac injuries with an AIS $\geq 3$ were not included because these subgroups of patients were admitted to the Department of Chest Surgery for surgical interventions. The cardiac specific group included patients with arrhythmia or patients with an increase in cardiac markers without significant changes in other vital signs, previously abnormal echocardiography results, cardiac symptoms such as chest pain, and previous heart disease history such as acute coronary syndrome and heart failure. The hemodynamic instability group included shock of unknown origin, ventricular tachycardia, and cardiac arrest.

\section{Abnormal Echocardiography findings}

All TTE results were categorized as either a positive or negative result. Positive findings were defined as any one of following findings: ejection fraction of $\leq 40 \%$ [8], moderate or more severe dysfunction of the left ventricle, moderate or more severe heart valve regurgitation, and akinesia of heart wall.

\section{Statistical analysis}

All statistical analysis was performed using IBM SPSS Statistics Version 20 (IBM, Armonk, NY, USA). Cross-matching, $t$ test, and analysis of variance were used depending on the variables. A $p<0.05$ was considered statistically significant.

\section{Results}

\section{Study population}

A total of 209 echocardiography examinations were performed on 187 general surgery patients in ICU during the study period. After excluding 3 cadaver donors, 184 patients and 206 examinations were used in this study. For analyzing indications for an echocardiography, 10 patients with 12 examinations that did not belong to any 1 of 3 indications were excluded from the analysis (Figure 1).

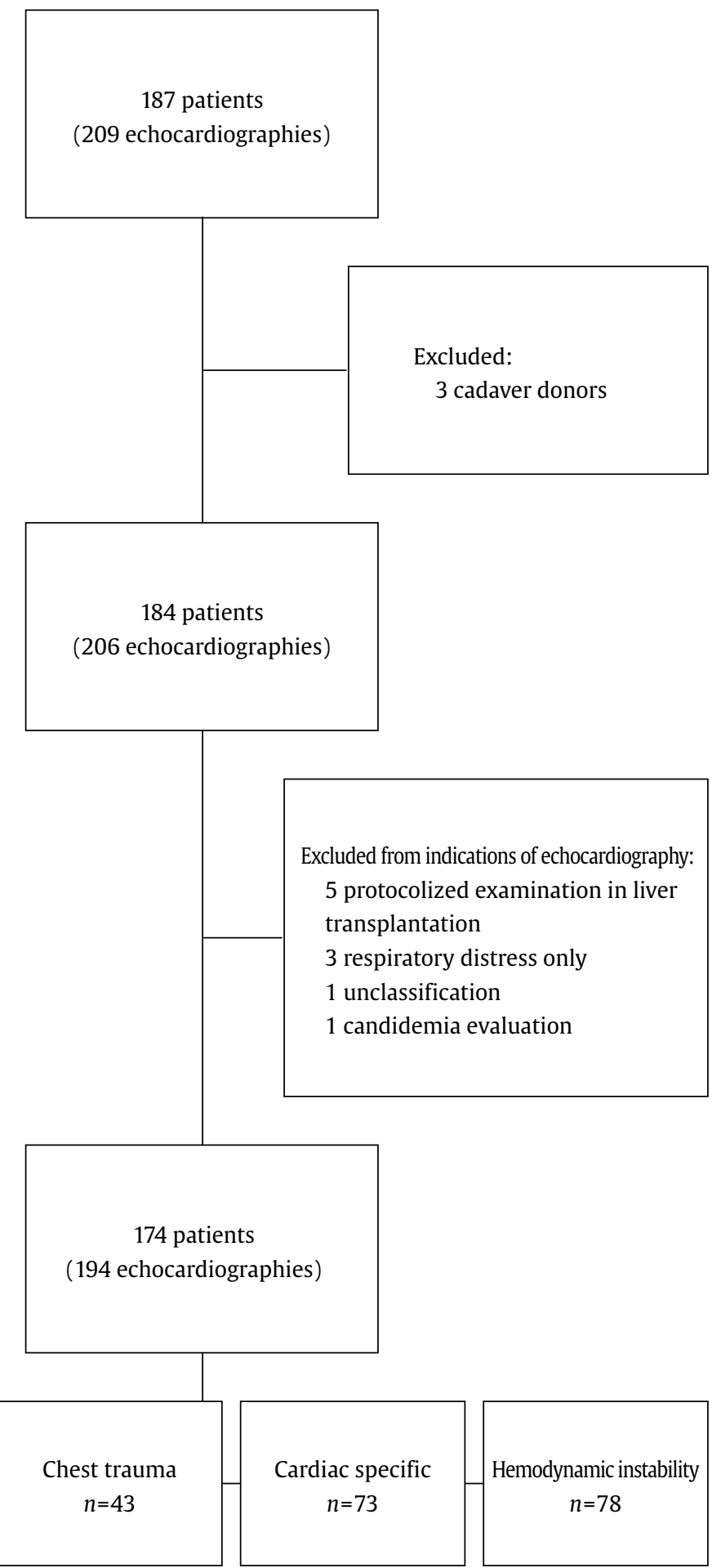

Figure 1. Flow diagram of the study population. 
Table 1. Patient characteristics.

\begin{tabular}{|c|c|}
\hline Variable & Value \\
\hline Patients $(n)$ & 184 \\
\hline Echocardiographies & 206 \\
\hline ICU admissions & 207 \\
\hline Age (y) & $65.8 \pm 15.2$ \\
\hline \multicolumn{2}{|l|}{ Gender } \\
\hline Male & $118(64.1)$ \\
\hline Female & $66(35.9)$ \\
\hline \multicolumn{2}{|l|}{ Reason for hospital admission } \\
\hline Trauma & $52(28.3)$ \\
\hline Acute abdomen & $69(37.5)$ \\
\hline Malignancy & $27(14.7)$ \\
\hline Benign disease & $35(19.6)$ \\
\hline \multicolumn{2}{|l|}{ Indication for ICU admission } \\
\hline Respiratory distress & $55(26.6)$ \\
\hline Hemodynamic instability & $100(48.3)$ \\
\hline Closed monitoring & $52(25.1)$ \\
\hline \multicolumn{2}{|l|}{$\operatorname{LOS}(\mathrm{d})$} \\
\hline ICU LOS & $14.9 \pm 19.2$ \\
\hline Hospital LOS & $35.6 \pm 34.0$ \\
\hline ICU-free days & $20.7 \pm 24.8$ \\
\hline \multicolumn{2}{|l|}{ In-hospital mortality } \\
\hline Yes & $61(33.2)$ \\
\hline No & $123(66.8)$ \\
\hline \multicolumn{2}{|l|}{ Mechanical ventilation } \\
\hline Yes & $142(77.2)$ \\
\hline No & $42(22.8)$ \\
\hline \multicolumn{2}{|l|}{ ECMO } \\
\hline Yes & $8(4.3)$ \\
\hline No & $176(95.7)$ \\
\hline \multicolumn{2}{|l|}{ Comorbidities } \\
\hline Hypertension & $92(50.0)$ \\
\hline Chronic obstructive pulmonary disease & $8(4.3)$ \\
\hline Coronary disease & $9(4.9)$ \\
\hline Renal disease & $6(3.3)$ \\
\hline Diabetes mellitus & $51(27.7)$ \\
\hline Hepatitis & $9(4.9)$ \\
\hline
\end{tabular}

Data are presented as mean \pm SD or $n(\%)$.

ICU = intensive care unit; LOS = length of stay; ECMO = extracorporeal membrane oxygenation.

\section{Patient characteristics}

The mean age was 65.8 years with a standard deviation of 15.2 years. The study population was predominantly male (male 118, 64.1\%). The reasons for hospital admission were: 52 patients due to trauma, 69 due to acute abdomen, 27 due to malignancy, and 35 due to other benign causes. The indications for ICU admission were: 55 due to respiratory distress, 100 due to hemodynamic instability, and 52 for close monitoring. The average ICU length of stay (LOS) was 14.9 days with standard deviation of 19.2 days. Out of 184 patients, there were 61 inhospital mortalities (Table 1).

\section{TTE}

Among the 184 patients who had TTE in ICU, 165 patients had TTE only once, 16 patients twice, and 3 patients had TTE 3 times. After excluding the 10 patients (12 echocardiographic examinations) who did not fall into the (1) chest trauma; (2) cardiac specific pathology; or (3) hemodynamic instability groups, there were a total of 194 echocardiography examinations performed (Figure 1). Indications for these 194 examinations consist of: 43 cases of chest trauma, 73 cases of cardiac specifics, and 78 cases of hemodynamic instability. Out of these 194 examinations, 45 examinations showed abnormal findings (Table 2).

Table 2. Characteristics of echocardiography examinations.

\begin{tabular}{ll}
\hline Variable & Value \\
\hline Echocardiographies/patient & \\
1 & $165(89.7)$ \\
2 & $16(8.7)$ \\
3 & $3(1.6)$ \\
Indication for echocardiography & \\
Chest trauma & $43(22.2)$ \\
Cardiac specific & $73(37.6)$ \\
Hemodynamic instability & $78(40.2)$ \\
\hline Positive result for the echocardiography & \\
Yes & $45(23.2)$ \\
No & $149(76.8)$ \\
\hline
\end{tabular}

Data are presented as $n$ (\%).

\section{Clinical characteristics according to TTE results}

Patients who had a positive echocardiography finding showed a higher mean age. In assessing the reason for hospital admission, the trauma patient group had the lowest rate of 
positive findings: $5.8 \%$ of the trauma group, $24.6 \%$ of the acute abdomen group, $29.6 \%$ of the malignancy group, and $27.8 \%$ of the benign group showed positive echocardiography findings.

In the hemodynamic instability and respiratory distress group significantly higher positive findings were observed compared with the close monitoring group, when classified according to the ICU admission indications (27.0\%, 18.2\%, 3.8\%, $p=0.03$ ).

In terms of application of ECMO, 5 of the 38 patients in the positive echocardiography findings group received ECMO while only 3 of the 146 patients in the negative echocardiography findings group received ECMO. Gender and length of stay were not significantly associated with TTE result (Table 3).

\section{Association with indications for echocardiography}

Positive findings for TTE were significantly associated with indications for TTE as $30.1 \%$ of the cardiac specific group and $25.6 \%$ of the hemodynamic instability group showed a positive TTE result, while only $7.0 \%$ of the chest trauma group showed abnormal TTE findings $(p=0.018)$. The "cardiac specifics" group and the "hemodynamic instability" group showed significantly higher in-hospital and ICU mortality than the chest trauma group $(p<0.001)$. Hospital or ICU length of stay were not significantly associated with the indication for an echocardiography (Table 4).

\section{Mortality}

In addition to the relationship between mortality and indication for TTE, the age of the patient, the reason for hospital admission, and the indication for ICU admission were
Table 3. Association between TTE findings and other clinical characteristics.

\begin{tabular}{lccc}
\hline \multirow{2}{*}{ Variable } & \multicolumn{3}{c}{ TTE findings } \\
\cline { 2 - 4 } & Positive & Negative & $p$ \\
\hline Age (y) & $70.3 \pm 15.7$ & $64.6 \pm 15.0$ & 0.041 \\
Gender & & & 0.318 \\
Male & $27(22.9)$ & $91(77.1)$ & \\
Female & $11(16.7)$ & $55(83.3)$ & \\
Reason for hospital & & & 0.01 \\
admission & & & \\
Trauma & $3(5.8)$ & $49(94.2)$ & \\
Acute abdomen & $17(24.6)$ & $52(75.4)$ & \\
Malignancy & $8(29.6)$ & $19(70.4)$ & \\
Benign disease & $10(27.8)$ & $26(72.2)$ & \\
Indication for ICU & & & 0.003 \\
admission (\%) & & & \\
Respiratory distress & $10(18.2)$ & $45(81.8)$ & \\
Hemodynamic instability & $27(27.0)$ & $73(73.0)$ & \\
Closed monitoring & $2(3.8)$ & $50(96.2)$ & \\
\hline Length of stay (d) & & & \\
ICU & $19.0 \pm 26.0$ & $13.9 \pm 16.9$ & 0.257 \\
Hospital & $36.4 \pm 39.3$ & $35.4 \pm 32.6$ & 0.876 \\
\hline
\end{tabular}

Data are presented as mean \pm SD or $n(\%)$.

TTE = transthoracic echocardiography; ICU = intensive care unit.

Table 4. Clinical outcomes and indications for an echocardiography.

\begin{tabular}{|c|c|c|c|c|}
\hline \multirow{2}{*}{ Variable } & \multicolumn{2}{|c|}{ Echocardiography Indication } & \multirow[b]{2}{*}{$\mathrm{HI}$} & \multirow[b]{2}{*}{$p$} \\
\hline & Chest trauma & Cardiac specific & & \\
\hline TTE abnormal findings & & & & 0.018 \\
\hline Yes & $3(7.0)$ & $22(30.1)$ & $20(25.6)$ & \\
\hline No & $40(93.0)$ & $51(69.9)$ & $58(74.4)$ & \\
\hline ICU LOS (d) & $9.6 \pm 17.3$ & $17.5 \pm 21.4$ & $16.2 \pm 19.1$ & 0.106 \\
\hline Hospital LOS (d) & $24.6 \pm 29.8$ & $40.3 \pm 35.0$ & $37.6 \pm 36.0$ & 0.057 \\
\hline ICU mortality & & & & 0.001 \\
\hline Yes & $5(11.6)$ & $22(30.1)$ & $33(42.3)$ & \\
\hline No & $38(88.4)$ & $51(69.9)$ & $45(57.7)$ & \\
\hline In-hospital mortality & & & & $<0.001$ \\
\hline Yes & $5(11.6)$ & $29(39.7)$ & $39(50.0)$ & \\
\hline No & $38(88.4)$ & $44(60.3)$ & $39(50.0)$ & \\
\hline
\end{tabular}

Data are presented as $n(\%)$ or mean \pm SD.

$\mathrm{HI}=$ hemodynamic instability; TTE = transthoracic echocardiography; LOS = length of stay; ICU = intensive care unit. 
Table 5. Association between mortality and clinical characteristics.

\begin{tabular}{|c|c|c|c|c|c|c|}
\hline \multirow{2}{*}{ Variable } & \multicolumn{3}{|c|}{ ICU mortality } & \multicolumn{3}{|c|}{ In-hospital mortality } \\
\hline & Yes & No & $p$ & Yes & No & $p$ \\
\hline Age $(y)$ & $68.9 \pm 13.1$ & $64.3 \pm 16.0$ & 0.052 & $69.6 \pm 12.8$ & $63.7 \pm 16.1$ & 0.007 \\
\hline Gender & & & 0.969 & & & 0.992 \\
\hline Male & $39(33.1)$ & $79(66.9)$ & & $43(36.4)$ & $75(63.6)$ & \\
\hline Female & $22(33.3)$ & $44(66.7)$ & & $24(36.4)$ & $42(63.6)$ & \\
\hline Reason for hospital admission & & & 0.001 & & & 0.001 \\
\hline Trauma & $8(15.4)$ & $44(84.6)$ & & $8(15.4)$ & $44(84.6)$ & \\
\hline Acute abdomen & $26(37.7)$ & $43(62.3)$ & & $28(40.6)$ & $41(59.4)$ & \\
\hline Malignancy & $10(37.0)$ & $17(63.0)$ & & $14(51.9)$ & $13(48.1)$ & \\
\hline Benign disease & $17(47.2)$ & $19(52.8)$ & & $17(47.2)$ & $19(52.8)$ & \\
\hline Indication for ICU admission & & & 0.031 & & & 0.009 \\
\hline Respiratory distress & $13(23.6)$ & $42(76.4)$ & & $19(34.5)$ & $36(65.5)$ & \\
\hline Hemodynamic instability & $48(48.0)$ & $52(52.0)$ & & $49(49.0)$ & $51(51.0)$ & \\
\hline Closed monitoring & $10(19.2)$ & $42(80.8)$ & & $11(21.2)$ & $41(78.8)$ & \\
\hline Indication for echocardiography & & & 0.001 & & & $<0.001$ \\
\hline Chest trauma & $5(11.6)$ & $38(88.4)$ & & $5(11.6)$ & $38(88.4)$ & \\
\hline Cardiac specific & $22(30.1)$ & $51(69.9)$ & & $29(39.7)$ & $44(60.3)$ & \\
\hline Hemodynamic instability & $33(42.3)$ & $45(57.7)$ & & $39(50.0)$ & $39(50.0)$ & \\
\hline
\end{tabular}

Data are presented as mean \pm SD or $n(\%)$.

ICU = intensive care unit.

also significantly associated with hospital mortality. Among the 3 indications for ICU admission, the hemodynamic instability group showed the highest hospital mortality (Table 5).

\section{Discussion}

An echocardiography is a test with many strengths attributed to its non-invasiveness, rapid real-time testing, portability of ultrasound equipment, and absence of exposure to radiation [9]. It is usually performed after the initial cardiology examinations, and its effectiveness, and widespread use is evident in patients attending outpatient clinics, and general wards. Echocardiographies are also used to evaluate perioperative cardiopulmonary risk in surgical patients $[6,10]$. There has been an increased use of echocardiography in ICUs and it is now an indispensable tool in evaluating critically ill patients [2,11]. An echocardiography serves many purposes for patients in ICUs. It is currently being used for assessing the severity of cardiac dysfunction, intravascular volume status, response to treatment in hypotensive patients, patients with chest pain, and patients after cardiac trauma or cardiac arrest
$[12,13]$. Guidelines for the use of echocardiography in ICUs have been proposed, but it seemed inadequate to recommend guidelines to be used specifically in ICUs [3]. However, critical general surgery patients may differ from other patients in ICU in the use of echocardiography, because postoperative wounds or dressings may limit the positioning of the ultrasound device, thus limiting the test window. There are many studies on the efficacy of the echocardiography in ICUs, but data is scarce when it comes to exclusively studying general surgery patients in ICU. Thus, this study was conducted using data (2014-2019) from only general surgery patients in ICU to seek appropriate indications and determine the usefulness of echocardiographies for the critical general surgery patients.

Many previous studies have demonstrated the usefulness of an echocardiography in the critical setting as a rescue and monitoring tool. In 2020, in a study in Canada of 274 general surgery patients in the ICU where TEE was used, reported that the common indications for TEE were hemodynamic instability (45.2\%) and cardiac arrest (20.1\%) [14]. Another study published in the USA in 2020 reported that in ICU patients (cardiac medical and surgical) the most common indication for a rescue point of care ultrasound examination was hypotension (49\%) 
[15]. Echocardiographies were recommended in Europe in 2014 as a useful modality for evaluating the underlying causes of hemodynamic instability [16]. That is, general indications for echocardiography in ICUs are (1) rescue diagnostic modality for hemodynamic instability including cardiac arrest, (2) monitoring tool for cardiac function, and (3) perioperative risk assessment for the cardiovascular deterioration of surgical patients, especially for the patients with cardiac disease history or abnormal findings on previous basic cardiac studies. The authors classified the indication for an echocardiography into 3 simplified indications based on the clinical and practical situation in the critical setting.

The first indication, chest trauma, is an important clinical event that requires an echocardiography. In 2012, the Eastern Association for the Surgery of Trauma updated practice management guidelines for recommending diagnostic echocardiographies for patients with blunt chest injury, and unexplained hypotension or arrhythmia [17]. Incidence of cardiac injury is rarer than injury to the pulmonary organ in patients with chest injury. Clinical manifestations of cardiac injury may be subtle in the early clinical period, and detection of cardiac injury may be difficult during that time period with physical examination, cardiac enzyme, electrocardiography, focused assessment sonography for trauma and even with chest $\mathrm{CT}$.

The echocardiographies were performed on patients with blunt chest injury using similar indications such as chest injury with an AIS 3 or above, circulatory shock of unclear origin, and as a short-term follow-up tool in case of ambiguous findings on initial chest x-ray or CT. Positive echocardiography findings were determined in only $7 \%$ of patients in the chest trauma group, which was significantly lower than the 2 remaining indication groups. With this significantly lower rate of positive findings, it is possible that most injured patients were healthy prior to the incidents, and less likely to have had comorbidities. It was also supposed that actual cardiac injury was rare per se in chest trauma patients. However, in a study by Rozycki et al [18] including 1,540 patients with chest injury, echocardiographies were reported to be accurate when used for the immediate evaluation of hypotensive patients with blunt chest trauma [18].

The second indication, hemodynamic instability, accounted for $40 \%$ of the total number of echocardiography examinations in this current study, and patients in this group showed the highest mortality (50\% hospital mortality of which $42 \%$ was ICU mortality). Patients with any kind of circulatory shock, need for assessment of fluid resuscitation response, cardiac arrest, life threatening arrhythmia, or very severe respiratory distress of unknown origin belonged to this group. The highest mortality in this group may be explained with very high severity of these clinical pathologies. The basic performance of echocardiographies include functional assessment, in addition to structural imaging. These capabilities make echocardiography a very useful tool to determine the reason for circulatory shock and assess the response to the treatment. Vincent et al [2] reported that repeated echocardiography is necessary, and effective for assessing the impact of treatments on cardiac function in the critical setting [2].

The third indication, the need for TEE in this study was called "cardiac specific." This was the patient group who had a stable hemodynamic status with previous abnormal findings of cardiac specific tests or history of heart diseases, including perioperative assessment of cardiovascular risk in general surgery ICU patients. Use of echocardiographies in the perioperative setting can be divided into either that of cardiac surgery or that of non-cardiac major surgery. This current study did not include any patients who had a perioperative echocardiography following cardiac surgery. Even for the patients undergoing non-cardiac major surgery, postoperative cardiopulmonary complications frequently place these patients in the high-risk group; this may be due to hypovolemia, hemorrhage, sepsis, or acute cardiac dysfunction. It is important to identify the patients who have significant risk of major cardiopulmonary adverse events in the perioperative period. The impact of a perioperative echocardiography examination on patient survival in the high-risk surgical population with multiple comorbidities has previously been reported [11,19].

There were several limitations in this study. Firstly, and most importantly, there was no consideration of the impact of echocardiography findings on the clinical decision-making process. Echocardiography findings have previously been reported to lead to changes in diagnosis and management of circulatory shock $[20,21]$. The current study only showed that there was an association between an echocardiography indication and the number of cases with positive echocardiography findings, in addition to indirect clinical outcomes such as mortality and length of stay. To further determine the impact and usefulness of the examination results, one should explore how echocardiography results influence clinical decisions and interventions. In a European survey study, the authors reported between $26 \%$ to $50 \%$ of the cases had changes in therapeutic strategy after undertaking echocardiographies [22]. Secondly, this was a retrospective study with limited medical records. For example, there was some difficulty in identifying detailed indications in the medical records because they were often omitted by the surgeons who requested the echocardiography examination. Thirdly, transesophageal echocardiography examinations were not included in the medical records. As mentioned above, hemodynamic monitoring with repeated echocardiographies is one of the most important roles of echocardiography, but there 
were a few cases of repeated examination in the data. Fourthly, the chest trauma group included injured patients with the chest AIS 3 or above. The patients of this group had variable clinical manifestations with wide ranges of measurement. This heterogeneous nature of severity could not be reflected appropriately in the results. Finally, there was some ambiguity in the indications for the groups.

However, despite these limitations, this study holds its value in that it ascertained the appropriate indications, and usefulness of echocardiographies in general surgery critical patients.

\section{Conclusion}

The presence of cardiac pathology and hemodynamic instability may be appropriate indications for performing TTE on general surgery patients in ICU; further studies are required.

\section{Conflicts of Interest}

No potential conflict of interest relevant to this article was reported.

\section{References}

[1] Otto CM. Textbook of Clinical Echocardiography, 6th ed. Philadelphia (PA): Elsevier; 2018. 127 p.

[2] Vincent JL, Rhodes A, Perel A, Martin GS, Rocca GD, Vallet B, et al. Clinical review: Update on hemodynamic monitoring - a consensus of 16 . Crit Care 2011;15(4):229.

[3] Levitov A, Frankel HL, Blaivas M, Kirkpatrick AW, Su E, Evans D, et al. Guidelines for the appropriate use of bedside general and cardiac ultrasonography in the evaluation of critically ill patients-part II: Cardiac ultrasonography. Crit Care Med 2016; 44(6):1206-27.

[4] Papolos A, Narula J, Bavishi C, Chaudhry F, Sengupta P. U.S. hospital use of echocardiography. Insights from the Nationwide Inpatient Sample. J Am Coll Cardiol 2016;67(5):502-11.

[5] Schmidt S, Dieks J-K, Quintel M, Moerer O. Critical care echocardiography as a routine procedure for the detection and early treatment of cardiac pathologies. Diagnostics (Basel) 2020;10(9):671.
[6] Douglas PS, Garcia MJ, Haines DE, Lai WW, Manning WJ, Patel AR, et al. ACCF/ASE/AHA/ASNC/HFSA/HRS/ SCAI/SCCM/SCCT/SCMR 2011 Appropriate use criteria for echocardiography. J Am Coll Cardiol 2011;57(9):1126-66.

[7] Beaulieu Y, Marik PE. Bedside ultrasonography in the ICU: part 1. Chest 2005;128(2):881-95.

[8] Yancy CW, Jessup M, Bozkurt B, Butler J, Casey DE Jr, Drazner MH, et al. 2013 ACCF/AHA guideline for the management of heart failure: A report of the American College of Cardiology Foundation/American Heart Association Task Force on practice guidelines. J Am Coll Cardiol 2013;62(16):e147-239.

[9] Casaroto E, Mohovic T, Pinto LM, Lara TR. Bedside echocardiography in critically ill patients. Einstein 2015;13(4):644-6.

[10] Jasudavisius A, Arellano R, Martin J, McConnell B, Bainbridge D. A systematic review of transthoracic and transesophageal echocardiography in noncardiac surgery: Implications for pointofcare ultrasound education in the operating room. Can J Anaesth 2016;63(4):480-7.

[11] Cholley BP, Vieillard-Baron A, Mebazaa A. Echocardiography in the ICU: Time for widespread use! Intensive Care Med 2005;32(1):9-10.

[12] Arntfield RT, Millington SJ. Point of care cardiac ultrasound applications in the emergency department and intensive care unit-A review. Curr Cardiol Rev 2012;8(2):98-108.

[13] Haji DL, Royse A, Royse CF. Review article: Clinical impact of noncardiologist-performed transthoracic echocardiography in emergency medicine, intensive care medicine and anaesthesia. Emerg Med Australas. 2013;25(1):4-12

[14] Arntfield R, Lau V, Landry Y, Priestap F, Ball I. Impact of critical care transesophageal echocardiography in medical-surgical ICU patients: Characteristics and results from 274 consecutive examinations. J Intensive Care Med 2020;35(9):896-902.

[15] Lu SY, Dalia AA, Cudemus G, Shelton KT. Rescue echocardiography/ Ultrasonography in the management of combined cardiac surgical and medical patients in a cardiac intensive care unit. J Cardiothorac Vasc Anesth 2020;34(10):2682-8.

[16] Cecconi M, De Backer D, Antonelli M, Beale R, Bakker J, Hofer C, et al. Consensus on circulatory shock and hemodynamic monitoring. Taskforce of the European society of intensive care medicine. Intensive Care Med 2014;40(12):1795-815.

[17] Clancy K, Velopulos C, Bilaniuk JW, Collier B, Crowley W, Kurek S, et al. Screening for blunt cardiac injury: An Eastern Association for the Surgery of Trauma practice management guideline. J Trauma and Acute Care Surgery 2012;73(5 Suppl 4):S301-6.

[18] Rozycki G, Ballard R, Feliciano D, Schmidt J, Pennington S. Surgeon performed ultrasound for the assessment of truncal injuries: Lessons learned from 1540 patients. Ann Surg 1998;228(4):557-67.

[19] Staudt GE, Shelton K. Development of a rescue echocardiography protocol for noncardiac surgery patients. Anesth Analg 2019;129(2):e37-40.

[20] Stanko LK, Jacobsohn E, Tam JW, De Wet CJ, Avidan M. Transthoracic echocardiography: impact on diagnosis and management in tertiary care intensive care units. Anaesth Intensive Care 2005;33(4):492-6.

[21] Orme RM, Oram MP, McKinstry CE. Impact of echocardiography on patient management in the intensive care unit: An audit of district general hospital practice. Br J Anaesth 2009;102(3):340-4.

[22] Balzer F, Trauzeddle RF, Ertmer M, Erb J, Heringlake M, Groesdonk HV, et al. Utilization of echocardiography in intensive care units: Results of an online survey in Germany. Minerva Anestesiologica 2019;85(3):263-70. 